\title{
SOME STEFFENSEN-TYPE ITERATIVE SCHEMES FOR THE APPROXIMATE SOLUTION OF NONLINEAR EQUATIONS
}

\author{
FAROOQ AHMED SHAH, MUHAMMAD ASLAM NOOR, MUHAMMAD WASEEM, \\ AND EHSAN UL HAQ
}

Received 11 December, 2018

\begin{abstract}
In this paper, we suggest some new and efficient iterative methods for solving nonlinear equations $f(x)=0$. These methods are free from derivatives having high order of convergence. We also give some examples to illustrate the efficiency of these methods. Finally, numerical tests confirm the theoretical results and allow us to compare these variants with the classical Steffensen's method. These new methods can be considered as alternative of existing derivative-free methods.
\end{abstract}

2010 Mathematics Subject Classification: 30C30; 34A34; 34G20

Keywords: iterative method, approximate solution, numerical results, convergence

\section{INTRODUCTION}

Finding the approximate solution of the nonlinear equation $f(x)=0$ is one of the basic problems which frequently occurs in scientific work of various fields [1-13]. Due to the higher order of the nonlinear equations and the involvement of transcendental functions, analytical methods cannot be employed and therefore, it is only possible to obtain approximate solutions by relying on numerical methods based on iteration procedure. There are several methods in the literature for the computation of the root of the nonlinear equations. Newton method is well known classical method which is described as:

$$
x_{n+1}=x_{n}-\frac{f\left(x_{n}\right)}{f^{\prime}\left(x_{n}\right)}, n=0,1,2, \ldots
$$

This method possesses quadratic convergence in the neighborhood of simple root $r$. This method is not applicable when the derivative of any function is not defined at initial or any stage of computations. There are also some interval based initial methods but have slow convergence towards the solution with additional drawbacks.

The work of Farooq Ahmed Shah is supported by the Higher Education Commission of Pakistan through Grant 5418/Federal/NRPU/ RD/HEC/2016, and in part by the COMSATS University, Islamabad (CUI) through under Grant 16-75/CRGP/CIIT/ATK/17/1141. 
Some time we face the complicated problems for whom the calculation of derivatives are more complicated to compute. Some of the problems of such type are listed here.

(1) $f(x)=\cos (x)+\sin (2 x) \sqrt{1-2 x^{2}}+\sin \left(x^{2}\right)+x^{14}+x^{3} \ln (x)+12$

(2) $f(x)=\left(1+x^{3}\right) \cos \left(x^{2}\right)+\sqrt{1-x^{2}}-2(9 \sqrt{2}+7 \sqrt{3}) x^{4}$

(3) $f(x)=\frac{1}{x}-|x|$.

Such kind of problems need derivative-free iterative methods for obtaining the approximate solutions. In the last few years, researchers have established the idea of removing derivatives from the main recurrence relations in order to escape defining new functions involving derivatives and tried to calculate iterations only by using the function that describes the problem. It is tried to preserve the order of convergence of the method after using the approximation of the involved derivatives. Steffensen $[5,13]$ introduced such type of method in which he used the approximation of derivative as:

$$
f^{\prime}\left(x_{n}\right) \approx \frac{f\left(x_{n}+f\left(x_{n}\right)-f\left(x_{n}\right)\right.}{f\left(x_{n}\right)}
$$

Using this approximation, Steffensen $[5,13]$ modified the Newton method as a derivative-free method:

$$
x_{n+1}=x_{n}-\frac{\left[f\left(x_{n}\right)\right]^{2}}{f\left(x_{n}+f\left(x_{n}\right)\right)-f\left(x_{n}\right)}, n=0,1,2, \ldots
$$

This method is known as Steffensen's iterative method for solving nonlinear equations. This method has quadratic convergence. Steffensen's method is free from any derivative of the function and have great importance because sometimes the applications of iterative methods which depend upon derivatives are restricted in engineering and other fields.

The rest of this paper is organized as follows. In Section 2, we describe our methods that are free from derivatives and have better convergence rate than Steffensen's method. In Section 3, we establish the convergence order of these methods. Finally, in Section 4 different numerical tests confirm the theoretical results and allow us to compare these methods with the existing methods.

\section{CONSTRUCTION OF DERIVATIVE-FREE ITERATIVE TECHNIQUES}

In this section, we derive some new iterative techniques for solving nonlinear equations without using the derivative of the function to obtain such type of iterative methods. We use approximation of the first derivatives of the function to obtain such type of iterative methods.

Let us approximate the first derivative of the function $f(x)$ by

$$
f^{\prime}(x)=\frac{f(x+b f(x))-f(x)}{b f(x)}, \because b \in \mathbb{R} \text { and } b \neq 0 .
$$

Now we use this approximation in well known Newton method $[3,13]$ and obtain the following derivative-free iterative method for solving nonlinear equations as: 
Algorithm 2.1. For a given $x_{0}$, find the approximate solution $x_{n+1}$ by the iterative scheme:

$$
x_{n+1}=x_{n}-\frac{b\left[f\left(x_{n}\right)\right]^{2}}{f\left(x_{n}+b f\left(x_{n}\right)\right)-f\left(x_{n}\right)}, n=0,1,2, \ldots
$$

For different values of $b$, we obtain classes of iterative schemes without involving derivatives. If we select, $b=1$, then Algorithm 2.1 reduces to the well known Steffensen's method $[5,13]$. Our aim is to modify higher order methods as derivative-free method preserving their order of convergence. Halley method is a well known cubic convergent method $[5,13]$ involving higher derivatives. This method has cubic convergence and can be derived by various techniques $[5,12,13]$. Halley method is described as:

Algorithm 2.2. For a given $x_{0}$, find the approximate solution $x_{n+1}$ by the iterative scheme:

$$
x_{n+1}=x_{n}-\frac{2 f\left(x_{n}\right) f^{\prime}\left(x_{n}\right)}{2\left[f^{\prime}\left(x_{n}\right)\right]^{2}-f\left(x_{n}\right) f^{\prime \prime}\left(x_{n}\right)}, n=0,1,2, \ldots
$$

This method involves second derivative of the function for solving nonlinear equations. We will use the approximation of the first and second derivatives of function $f(x)$ as:

$$
\begin{gathered}
f^{\prime}\left(x_{n}\right) \approx \frac{f\left(x_{n}+b f\left(x_{n}\right)\right)-f\left(x_{n}-b f\left(x_{n}\right)\right)}{2 b\left[f\left(x_{n}\right)\right]}, \\
f^{\prime \prime}\left(x_{n}\right) \approx \frac{f\left(x_{n}+b f\left(x_{n}\right)\right)-2 f(x)+f\left(x_{n}-b f\left(x_{n}\right)\right)}{\left[b f\left(x_{n}\right)\right]^{2}} .
\end{gathered}
$$

Using the above approximations of derivatives in Algorithm 2.2, we obtain the following derivative-free iterative method as:

Algorithm 2.3. For a given $x_{0}$, find the approximate solution $x_{n+1}$ by the iterative scheme:

$$
x_{n+1}=x_{n}-\frac{b\left[f\left(x_{n}\right)\right]^{2} A\left(x_{n}\right)}{\left[A\left(x_{n}\right)\right]^{2}-2 f\left(x_{n}\right)\left[f\left(x_{n}+b f\left(x_{n}\right)\right)-2 f\left(x_{n}\right)+f\left(x_{n}-b f\left(x_{n}\right)\right)\right.},
$$

where

$$
A\left(x_{n}\right)=\left[f\left(x_{n}+b f\left(x_{n}\right)\right)-f\left(x_{n}-b f\left(x_{n}\right)\right)\right] \because b \in \mathbb{R} \text {, and } b \neq 0 .
$$

Algorithm 2.3 is a newly derived third order convergent derivative-free iterative method for solving nonlinear equations. Shah and Noor [12] suggested a fourth-order iterative method for solving nonlinear equations as:

Algorithm 2.4. For a given $x_{0}$, find the approximate solution $x_{n+1}$ by the iterative schemes:

$$
\begin{gathered}
y_{n}=x_{n}-\frac{2 f\left(x_{n}\right) f^{\prime}\left(x_{n}\right)}{2\left[f^{\prime}\left(x_{n}\right)\right]^{2}-f\left(x_{n}\right) f^{\prime \prime}\left(x_{n}\right)}, \\
x_{n+1}=y_{n}-\frac{2 f\left(y_{n}\right) f^{\prime}\left(x_{n}\right)}{2\left[f^{\prime}\left(x_{n}\right)\right]^{2}-f\left(x_{n}\right) f^{\prime \prime}\left(x_{n}\right)}, n=0,1,2, \ldots
\end{gathered}
$$


Algorithm 2.4 involves the second derivative of the function. We use the approximations of the first and the second derivative of function $f\left(x_{n}\right)$ and obtain the new iterative scheme by this modification as:

Algorithm 2.5. For a given $x_{0}$, find the approximate solution $x_{n+1}$ by the iterative schemes:

$$
\begin{gathered}
y_{n}=x_{n}-\frac{2 b A\left(x_{n}\right)}{\left[A\left(x_{n}\right)\right]^{2}-2 f\left(x_{n}\right)\left[f\left(x_{n}+b f\left(x_{n}\right)\right)-2 f\left(x_{n}\right)+f\left(x_{n}-b f\left(x_{n}\right)\right)\right.}, \\
x_{n+1}=y_{n}-\frac{2 b f\left(x_{n}\right) f\left(y_{n}\right)\left[A\left(x_{n}\right)\right]}{\left[A\left(x_{n}\right)\right]^{2}-2 f\left(x_{n}\right)\left[f\left(x_{n}+b f\left(x_{n}\right)\right)-2 f\left(x_{n}\right)+f\left(x_{n}-b f\left(x_{n}\right)\right)\right.} .
\end{gathered}
$$

Algorithm 2.5 is a fourth order convergent derivative-free iterative method for solving nonlinear equations.

Shah [11] suggested the following fourth-order iterative method for solving nonlinear equations.

Algorithm 2.6. For a given $x_{0}$, find the approximate solution $x_{n+1}$ by the iterative schemes:

$$
\begin{gathered}
y_{n}=x_{n}-\frac{2 f\left(x_{n}\right) f^{\prime}\left(x_{n}\right)}{\left.2\left[f^{\prime}\left(x_{n}\right)\right]^{2}-f\left(x_{n}\right) f^{\prime \prime}\left(x_{n}\right)\right)}, \\
x_{n+1}=y_{n}-\frac{4 f\left(y_{n}\right) f^{\prime}\left(x_{n}\right)}{\left.2\left[f^{\prime}\left(x_{n}\right)\right]^{2}-f\left(x_{n}\right) f^{\prime \prime}\left(x_{n}\right)\right)}-\frac{4 f\left(y_{n}\right) f^{\prime}\left(y_{n}\right)\left[f^{\prime}\left(x_{n}\right)\right]^{2}}{\left.\left[2\left[f^{\prime}\left(x_{n}\right)\right]^{2}-f\left(x_{n}\right) f^{\prime \prime}\left(x_{n}\right)\right)\right]^{2}} .
\end{gathered}
$$

This method also involves the derivatives of the function. We modify this method by using approximation of derivatives and obtain the following derivative-free iterative method.

Algorithm 2.7. For a given $x_{0}$, find the approximate solution $x_{n+1}$ by the iterative schemes:

$$
\begin{aligned}
y_{n}= & x_{n}-\frac{2 b\left[f\left(x_{n}\right)\right]^{2}\left[A\left(x_{n}\right)\right]}{\left[A\left(x_{n}\right)\right]^{2}-2 f\left(x_{n}\right)\left[f\left(x_{n}+b f\left(x_{n}\right)\right)-2 f\left(x_{n}\right)+f\left(x_{n}-b f\left(x_{n}\right)\right)\right.}, \\
x_{n+1}= & y_{n}-\frac{4 b f\left(x_{n}\right) f\left(y_{n}\right)\left[A\left(x_{n}\right)\right]}{\left[A\left(x_{n}\right)\right]^{2}-2 f\left(x_{n}\right)\left[f\left(x_{n}+b f\left(x_{n}\right)\right)-2 f\left(x_{n}\right)+f\left(x_{n}-b f\left(x_{n}\right)\right)\right.} \\
& -\frac{4\left[b f\left(x_{n}\right)\right]^{3}\left[A\left(x_{n}\right)\right]\left[f\left(y_{n}+b f\left(y_{n}\right)\right)-f\left(y-b f\left(y_{n}\right)\right)\right]}{\left[A\left(x_{n}\right)\right]^{2}-2 f\left(x_{n}\right)\left[f\left(x_{n}+b f\left(x_{n}\right)\right)-2 f\left(x_{n}\right)+f\left(x_{n}-b f\left(x_{n}\right)\right)\right]^{2}} .
\end{aligned}
$$

Algorithm 2.7 is a fourth order convergent derivative-free iterative method for solving nonlinear equations.

Noor [10] suggested the following two iterative methods for solving nonlinear equations which involve the first derivative of the function as:

Algorithm 2.8. For a given $x_{0}$, find the approximate solution $x_{n+1}$ by the iterative schemes:

$$
y_{n}=x_{n}-\frac{f\left(x_{n}\right)}{f^{\prime}\left(x_{n}\right)},
$$




$$
x_{n+1}=y_{n}-\frac{f\left(x_{n}\right)+h f\left(y_{n}\right)}{f^{\prime}\left(y_{n}\right)} .
$$

Now we use the suggested approximation in Algorithm 2.8 and obtain a derivativefree iterative method as:

Algorithm 2.9. For a given $x_{0}$, find the approximate solution $x_{n+1}$ by the iterative schemes:

$$
\begin{aligned}
& x_{n+1}=x_{n}-\frac{2 b\left[f\left(x_{n}\right)\right]^{2}}{f\left(x_{n}+b f\left(x_{n}\right)\right)-f\left(x_{n}-b f\left(x_{n}\right)\right)}, \\
& x_{n+1}=y_{n}-\frac{2 b f\left(y_{n}\right)\left[f\left(x_{n}\right)+h f\left(y_{n}\right)\right]}{f\left(y_{n}+b f\left(y_{n}\right)\right)-f\left(y_{n}-b f\left(y_{n}\right)\right)} .
\end{aligned}
$$

Similarly Noor [10] also suggested a method which involves derivatives:

Algorithm 2.10. For a given $x_{0}$, find the approximate solution $x_{n+1}$ by the iterative schemes:

$$
\begin{gathered}
y_{n}=x_{n}-\frac{f\left(x_{n}\right)}{f^{\prime}\left(x_{n}\right)}, \\
x_{n+1}=y_{n}-\frac{f\left(x_{n}\right)+2 h f\left(y_{n}\right)}{f^{\prime}\left(x_{n}\right)}+\frac{h f\left(y_{n}\right) f^{\prime}\left(y_{n}\right)}{[f(x)]^{2}} .
\end{gathered}
$$

Using the approximation in Algorithm 2.10 we obtain a derivative-free method as:

Algorithm 2.11. For a given $x_{0}$, find the approximate solution $x_{n+1}$ by the iterative schemes:

$$
\begin{gathered}
y_{n}=x_{n}-\frac{2 b\left[f\left(x_{n}\right)\right]^{2}}{f\left(x_{n}+b f\left(x_{n}\right)\right)-f\left(x_{n}-b f\left(x_{n}\right)\right)}, \\
x_{n+1}=y_{n}-\frac{2 b f\left(x_{n}\right)\left[f\left(x_{n}\right)+2 h f\left(y_{n}\right)\right]}{f\left(x_{n}+b f\left(x_{n}\right)\right)-f\left(x_{n}-b f\left(x_{n}\right)\right)}+\frac{h\left[f\left(y_{n}+b f\left(y_{n}\right)\right)-f\left(y_{n}-b f\left(y_{n}\right)\right)\right]}{2 b\left[f\left(x_{n}\right)\right]^{2}} .
\end{gathered}
$$

\section{CONVERGEnCE ANALysis}

In this section, we consider the convergence criteria of Algorithm 2.5 developed in Section 2. All other methods can be studied for the convergence in a similar manner.

Theorem 1. Let $\alpha$ be a simple zero of a sufficiently differentiable function $f: D \subset \mathbb{R} \rightarrow \mathbb{R}$ in an open interval D. If $x_{0}$ is sufficiently close to $\alpha$, then Algorithm 2.5 has fourth order convergence and satisfies the error equation

$$
e_{n+1}=\left[\left(c_{2}^{2}-c_{3}+c_{3} b^{2}\left(c_{1}\right)^{2}\right) e_{n}^{4}+O\left(e_{n}^{5}\right)\right] .
$$

Proof. Let $\alpha$ be the simple root of $f(x)$. Using Taylor series and expanding $f\left(x_{n}\right)$, $f\left(x_{n}-b f\left(x_{n}\right)\right)$ and $f\left(x_{n}+b f\left(x_{n}\right)\right)$, we obtain

$$
\begin{gathered}
f\left(x_{n}\right)=c_{1}\left[e_{n}+c_{2} e_{n}^{2}+c_{3} e_{n}^{3}+c_{4} e_{n}^{4}+c_{5} e_{n}^{5}+O\left(e_{n}^{6}\right)\right], \\
\left.f\left(x_{n}+b f\left(x_{n}\right)\right)=c_{1}\left(1+b c_{1}\right) e_{n}+3\left(c_{1}\right)^{2} b c_{2}+c_{1} c_{2}+\left(c_{1}\right)^{3} b^{2} c_{2}\right) e_{n}^{2}+O\left(e_{n}^{3}\right)
\end{gathered}
$$


and

$$
\left.f\left(x_{n}-b f\left(x_{n}\right)\right)=\left[-c_{1}\left(-1+b c_{1}\right) e_{n}+\left(3 c_{1}\right)^{2} b c_{2}+c_{1} c_{2}+\left(c_{1}\right)^{3} b^{2} c_{2}\right) e_{n}^{2}+O\left(e_{n}^{3}\right)\right],
$$

where

$$
c_{k}=\frac{f^{(k)}(p)}{f^{\prime}(p)}, k=2,3, \ldots, c_{1}=f^{\prime}(p), e_{n}=x_{n}-p .
$$

Now using (3.2) and (3.3), we obtain

$$
\begin{aligned}
& f\left(x_{n}+b f\left(x_{n}\right)\right)+f\left(x_{n}-b f\left(x_{n}\right)\right) \\
& \left.\quad=\left[2 b\left(c_{1}\right)^{2} e_{n}+6\left(c_{1}\right)^{2} b c_{2} e_{n}^{2}+4 b\left(c_{1}\right)^{2} c_{2}^{2}+8 b\left(c_{1}\right)^{2} c_{3}+2 c_{3}\left(c_{1}\right)^{4} b^{3}\right) e_{n}^{3}+O\left(e_{n}^{4}\right)\right] .
\end{aligned}
$$

Using (3.1), (3.2), (3.3) and (3.4) in Algorithm 2.5, we have

$$
\begin{aligned}
& x_{n}- \\
& \frac{2 b\left[f\left(x_{n}\right)\right]^{2}\left[f\left(x_{n}+b f\left(x_{n}\right)\right)-f\left(x_{n}-b f\left(x_{n}\right)\right)\right]}{\left[f\left(x_{n}+b f\left(x_{n}\right)\right)-f\left(x_{n}-b f\left(x_{n}\right)\right)\right]^{2}-2 f\left(x_{n}\right)\left[f\left(x_{n}+b f\left(x_{n}\right)\right)-2 f\left(x_{n}\right)+f\left(x_{n}-b f\left(x_{n}\right)\right)\right.} \\
& =\alpha+\left[\left(c_{2}^{2}-c_{3}+c_{3} b^{2}\left(c_{1}\right)^{2}\right) e_{n}^{3}+O\left(e_{n}^{4}\right)\right] .
\end{aligned}
$$

Using above all equations and simple manipulations yield the following error equations

$$
e_{n+1}=\left[\left(c_{2}^{2}-c_{3}+c_{3} b^{2}\left(c_{1}\right)^{2}\right) e_{n}^{4}+O\left(e_{n}^{5}\right)\right] .
$$

This shows that Algorithm 2.5 has fourth order convergence.

\section{Numerical RESUlts}

In this section, we exhibit computational comparison of newly derived methods for some examples. All the examples are tested by using Maple 11. Stopping criteria used for the computational work are given as:

- $\left|x_{n}-x_{n-1}\right|<\varepsilon$,

- $\left|f\left(x_{n}\right)\right|<\varepsilon$, where $\varepsilon=10^{-64}$.

Example 1. We consider the nonlinear equation $f(x)=\cos (x)-x$, and the initial guess for all the methods is $x_{0}=2$. Results and comparison is shown in Table 1 and Table 2 for $b=1$ and $b=0.25$ respectively. 
TABLE 1. Comparison of methods for $b=1$.

\begin{tabular}{|c|c|c|c|c|}
\hline Method & IT & $x_{n}$ & $\left|x_{n}-x_{n-1}\right|$ & $\left|f\left(x_{n}\right)\right|$ \\
\hline SM & 6 & 0.7390848856 & $5.08170 e^{-22}$ & 0.0000000 \\
\hline Alg 2.5 & 3 & 0.7390848856 & $1.12139 e^{-3}$ & $6.8890 e^{-12}$ \\
\hline Alg 2.7 & 3 & 0.7390848856 & $4.63400 e^{-5}$ & $7.9041 e^{-9}$ \\
\hline Alg 2.9 & 5 & 0.7390848856 & $1.19850 e^{-20}$ & 0.0000000 \\
\hline Alg 2.11 & 3 & 0.7390848856 & $2.53515 e^{-23}$ & 0.0000000 \\
\hline
\end{tabular}

TABLE 2. Comparison of methods for $b=\frac{1}{4}$.

\begin{tabular}{|c|c|c|c|c|}
\hline Method & IT & $x_{n}$ & $\left|x_{n}-x_{n-1}\right|$ & $\left|f\left(x_{n}\right)\right|$ \\
\hline SM & 6 & 0.7390848856 & $5.08170 e^{-22}$ & 0.0000000 \\
\hline Alg 2.5 & 3 & 0.7390848856 & $1.29387 e^{-05}$ & $1.45143 e^{-8}$ \\
\hline Alg 2.7 & 3 & 0.7390848856 & $1.5601833 e^{-05}$ & $5.16302 e^{-6}$ \\
\hline Alg 2.9 & 6 & 0.7390848856 & $3.40895 e^{-16}$ & 0.0000000 \\
\hline Alg 2.11 & 6 & 0.7390848856 & $8.68285 e^{-23}$ & 0.0000000 \\
\hline
\end{tabular}

TABLE 3. Comparison of methods for $b=1$.

\begin{tabular}{|c|c|c|c|c|}
\hline Method & IT & $x_{n}$ & $\left|x_{n}-x_{n-1}\right|$ & $\left|f\left(x_{n}\right)\right|$ \\
\hline SM & 11 & 1.36523001478 & 0.00000000 & $2.31492 e^{-14}$ \\
\hline Alg 2.5 & 6 & 1.36523001478 & $2.6400 e^{-7}$ & $2.45754 e^{-17}$ \\
\hline Alg 2.7 & 4 & 1.36523001478 & $9.823 e^{-6}$ & $1.609915 e^{-14}$ \\
\hline Alg 2.9 & 8 & 1.36523001478 & 0.00000000 & $2.2193 e^{-24}$ \\
\hline Alg 2.11 & 7 & 1.36523001478 & 0.00000000 & $6.08207 e^{-27}$ \\
\hline
\end{tabular}

TABLE 4. Comparison of methods for $b=\frac{1}{4}$.

\begin{tabular}{|c|c|c|c|c|}
\hline Method & IT & $x_{n}$ & $\left|x_{n}-x_{n-1}\right|$ & $\left|f\left(x_{n}\right)\right|$ \\
\hline SM & 11 & 1.36523001478 & 0.0000000 & $2.31492 e^{-14}$ \\
\hline Alg 2.5 & 3 & 1.36523001478 & $8.17300 e^{-9}$ & $6.136728 e^{-14}$ \\
\hline Alg 2.7 & 3 & 1.36523001478 & $2.103500 e^{-7}$ & $6.671405 e^{-5}$ \\
\hline Alg 2.9 & 8 & 1.36523001478 & 0.0000000 & $2.5083 e^{-17}$ \\
\hline Alg 2.11 & 7 & 1.36523001478 & 0.0000000 & $5.0634 e^{-32}$ \\
\hline
\end{tabular}

Example 2. We consider the nonlinear equation $f(x)=x^{3}+4 x-10$. 
Table 3 and Table 4 depict the numerical results of Example 2. We use the initial guess $x_{0}=1.5$ for the computer program.

Example 3. Consider the nonlinear equation $f(x)=2 x^{3}-3 x^{2}$. We observe that its derivative becomes zero on the interval $\left[\frac{1}{2}, 2\right]$, so for this function we can use only derivative-free methods.

Table 5 and Table 6 depict the numerical results of Example 3. We use the initial guess $x_{0}=0.5$ for the computer program.

TABLE 5. Comparison of methods for $b=1$.

\begin{tabular}{|c|c|c|c|c|}
\hline Method & IT & $x_{n}$ & $\left|x_{n}-x_{n-1}\right|$ & $\left|f\left(x_{n}\right)\right|$ \\
\hline SM & 23 & 1.499999335 & $1.0449181 e^{-18}$ & 0.0000000 \\
\hline Alg 2.5 & 5 & 1.499999335 & $5.5190301 e^{-4}$ & $-3.4115 e^{-5}$ \\
\hline Alg 2.7 & 4 & 1.499999335 & $2.6258151 e^{-5}$ & $2.566 e^{-6}$ \\
\hline Alg 2.9 & 9 & 1.499999335 & $7.5387141 e^{-15}$ & 0.0000000 \\
\hline Alg 2.11 & 9 & 1.499999335 & $1.7219124 e^{-16}$ & 0.0000000 \\
\hline
\end{tabular}

TABLE 6. Comparison of methods for $b=\frac{1}{4}$.

\begin{tabular}{|c|c|c|c|c|}
\hline Method & IT & $x_{n}$ & $\left|x_{n}-x_{n-1}\right|$ & $\left|f\left(x_{n}\right)\right|$ \\
\hline SM & 23 & 1.499999335 & $1.0449181 e^{-18}$ & 0.0000000 \\
\hline Alg 2.5 & 4 & 1.499999335 & $2.3284121 e^{-6}$ & $-4.569 e^{-12}$ \\
\hline Alg 2.7 & 3 & 1.499999335 & $1.0355809 e^{-6}$ & $4.04686 e^{-9}$ \\
\hline Alg 2.9 & 7 & 1.499999335 & $4.6836141 e^{-16}$ & 0.0000000 \\
\hline Alg 2.11 & 7 & 1.499999335 & $2.6909900 e^{-16}$ & 0.0000000 \\
\hline
\end{tabular}

Example 4. Consider the nonlinear equation

$$
f(x)=\cos (x)+\sin (2 x) \sqrt{1-x^{2}}+\sin \left(x^{2}\right)+x^{14}+x^{3}+1 / 2 x
$$

with $x_{0}=-0.92$.

Table 7 and Table 8 show the numerical results of Example 4.

Example 5. Consider the nonlinear equation $f(x)=\frac{1}{x}-|x|$ with $x_{0}=0.7$.

Table 9 and Table 10 show the numerical results of Example 5. 
TABLE 7. Comparison of methods for $b=1$.

\begin{tabular}{|c|c|c|c|c|}
\hline Method & IT & $x_{n}$ & $\left|x_{n}-x_{n-1}\right|$ & $\left|f\left(x_{n}\right)\right|$ \\
\hline SM & 5 & -0.92577268 & $7.6591100 e^{-20}$ & 0.0000000 \\
\hline Alg 2.5 & 2 & -0.92577268 & $3.0216400 e^{-4}$ & $2.634 e^{-6}$ \\
\hline Alg 2.7 & 2 & -0.92577268 & $3.2210000 e^{-4}$ & $2.566 e^{-6}$ \\
\hline Alg 2.9 & 4 & -0.92577268 & $1.7934900 e^{-17}$ & 0.0000000 \\
\hline Alg 2.11 & 4 & -0.92577268 & $1.6139720 e^{-22}$ & 0.0000000 \\
\hline
\end{tabular}

TABLE 8. Comparison of methods for $b=\frac{1}{2}$.

\begin{tabular}{|c|c|c|c|c|}
\hline Method & IT & $x_{n}$ & $\left|x_{n}-x_{n-1}\right|$ & $\left|f\left(x_{n}\right)\right|$ \\
\hline SM & 5 & -0.92577268 & $7.6591100 e^{-20}$ & 0.0000000 \\
\hline Alg 2.5 & 2 & -0.92577268 & $2.2412000 e^{-4}$ & $5.647 e^{-7}$ \\
\hline Alg 2.7 & 2 & -0.92577268 & $5.0631000 e^{-4}$ & $4.327 e^{-8}$ \\
\hline Alg 2.9 & 4 & -0.92577268 & $1.0470550 e^{-4}$ & 0.0000000 \\
\hline Alg 2.11 & 5 & -0.92577268 & $8.3425000 e^{-27}$ & 0.0000000 \\
\hline
\end{tabular}

TABLE 9. Comparison of methods for $b=1$.

\begin{tabular}{|c|c|c|c|c|}
\hline Method & IT & $x_{n}$ & $\left|x_{n}-x_{n-1}\right|$ & $\left|f\left(x_{n}\right)\right|$ \\
\hline SM & 6 & 0.9999999932 & $3.2474947 e^{-18}$ & 0.0000000 \\
\hline Alg 2.5 & 4 & 1.00000027 & $5.9794800 e^{-4}$ & $1.5650 e^{-10}$ \\
\hline Alg 2.7 & 7 & 1.00000027 & $1.2535300 e^{-5}$ & $7.01990 e^{-7}$ \\
\hline Alg 2.9 & 16 & 1.00000027 & $1.2615400 e^{-25}$ & 0.0000000 \\
\hline Alg 2.11 & 13 & 1.00000027 & $2.7432108 e^{-21}$ & 0.0000000 \\
\hline
\end{tabular}

TABLE 10. Comparison of methods for $b=\frac{1}{2}$.

\begin{tabular}{|c|c|c|c|c|}
\hline Method & IT & $x_{n}$ & $\left|x_{n}-x_{n-1}\right|$ & $\left|f\left(x_{n}\right)\right|$ \\
\hline SM & 6 & 0.9999999932 & $3.2474947 e^{-18}$ & 0.000000 \\
\hline Alg 2.5 & 3 & 0.9999999932 & $8.8873000 e^{-5}$ & $9.88077 e^{-8}$ \\
\hline Alg 2.7 & 4 & 0.9999999932 & $5.4401360 e^{-5}$ & $7.25509 e^{-6}$ \\
\hline Alg 2.9 & 5 & 0.9999999932 & $7.8798800 e^{-14}$ & 0.0000000 \\
\hline Alg 2.11 & 6 & 0.9999999932 & $8.7039110 e^{-21}$ & 0.0000000 \\
\hline
\end{tabular}

\section{CONCLUSION}

In this article, we have used approximations with a parameter $b \in R$ for the first and higher derivatives in various known methods. These approximations generated 
the new derivative-free methods and also these newly derived methods preserved the same order of convergence. The theoretical results have been checked with some numerical examples. Comparison is also given to check the performance and efficiency of these iterative schemes.

\section{ACKNOWLEDGMENT}

The authors would like to thank the editor and the anonymous reviewers for their constructive comments and suggestions.

\section{REFERENCES}

[1] S. Amat and S. Busquier, " A two-step Steffensen's method under modified convergence conditions.” J. Math. Anal. Appl., vol. 324, p. 1084-1092, 2006, doi: 10.1016/j.jmaa.2005.12.078.

[2] I. K. Argyors, "On the Secant method for solving nonsmooth equations." J. Math. Anal. Appl., vol. 322, p. 146-157, 2006, doi: 10.1016/j.jmaa.2005.09.006.

[3] M. A. Hasan, "Derivative-free family of higher order root finding methods." Hyatt Regency Riverfront, 2009.

[4] J. H. He, "Variational iteration method-some recent results and new interpretations," J. Comp. Appl. Math., vol. 207, pp. 3-11, 2007, doi: 10.1016/j.cam.2006.07.009.

[5] L. Johnson and R. D. Riess, Numerical Analysis. MA: Addison-Wesley, $1977 . \quad$ doi: 10.1002/oca.4660080111.

[6] D. Le, "An efficient derivative-free method for solving nonlinear equations," TOMS, vol. 11, no. 37, pp. 250-262, 1985, doi: 10.1145/214408.214416.

[7] M. A. Noor, "New classes of iterative methods for nonlinear equations," Appl. Math. Comput., vol. 191, pp. 128-131, 2007, doi: 10.1016/j.amc.2007.02.098.

[8] M. A. Noor and F. A. Shah, "Variational iteration technique for solving nonlinear equations," $J$. Appl. Math. Comput., vol. 31, pp. 247-254, 2009, doi: 10.1007/s12190-008-0207-4.

[9] M. A. Noor, F. A. Shah, K. I. Noor, and E. Al-Said, "Variational iteration technique for finding multiple roots of nonlinear equations," Sci. Res. Essays., vol. 6, no. 6, pp. 1344-1350, 2011, doi: 10.5897/SRE10.1057.

[10] M. Noor, "Iterative methods for nonlinear equations using homotopy perturbation techniques." Appl. Math. Inf. Sci., vol. 4, no. 2, pp. 227-235, 2010.

[11] F. Shah, "Modified homotopy perturbation technique for the approximate solution of nonlinear equations." Chin. J. Math., vol. 2014, pp. 1-10, 2014, doi: 10.1155/2014/787591.

[12] F. Shah and M. Noor, "Some numerical methods for solving nonlinear equations by using decomposition technique." Appl. Math. Comput., vol. 251, pp. 378-386, 2015, doi: 10.1016/j.amc.2014.11.065.

[13] J. F. Traub, Iterative methods for solutions of equations. Printice Hall, New York, 1964. doi: 10.1137/1008130.

Authors' addresses

Farooq Ahmed Shah

(Corresponding author) Department of Mathematics, COMSATS University Islamabad, Attock Campus, Pakistan

E-mail address: farooghamdani@gmail.com 
Muhammad Aslam Noor

Department of Mathematics, COMSATS University Islamabad, Main Campus, Pakistan

E-mail address: noormas lamehotmail.com

Muhammad Waseem

Department of Mathematics, COMSATS University Islamabad, Vehari Campus, Pakistan

E-mail address: sattarmwaseem@gmail.com

Ehsan ul Haq

Department of Mathematics, University of Wah, Wah Cantt., Pakistan

E-mail address: ehsan.shah61@gmail.com 